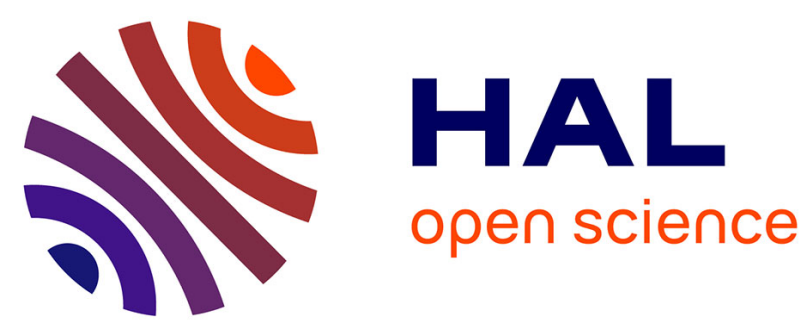

\title{
DIFFERENTIAL EXPLORATION OF COCHLEAR AND VESTIBULAR RECEPTORS BY INTERACTION OF ARTIFICIAL AND NATURAL STIMULI
}

\author{
R. Charlet de Sauvage, G. Dolivet, J.-P. Erre, J.-M. Aran
}

\section{To cite this version:}

R. Charlet de Sauvage, G. Dolivet, J.-P. Erre, J.-M. Aran. DIFFERENTIAL EXPLORATION OF COCHLEAR AND VESTIBULAR RECEPTORS BY INTERACTION OF ARTIFICIAL AND NATURAL STIMULI. Journal de Physique Colloques, 1990, 51 (C2), pp.C2-115-C2-118. 10.1051/jphyscol:1990227 . jpa-00230601

\section{HAL Id: jpa-00230601 https://hal.science/jpa-00230601}

Submitted on 1 Jan 1990

HAL is a multi-disciplinary open access archive for the deposit and dissemination of scientific research documents, whether they are published or not. The documents may come from teaching and research institutions in France or abroad, or from public or private research centers.
L'archive ouverte pluridisciplinaire HAL, est destinée au dépôt et à la diffusion de documents scientifiques de niveau recherche, publiés ou non, émanant des établissements d'enseignement et de recherche français ou étrangers, des laboratoires publics ou privés. 
COLLOQUE DE PHYSIQUE

Colloque C2, supplément au $\mathrm{n}^{\circ} 2$, Tome 51, Février 1990

ler Congrès Français d'Acoustique 1990

\title{
DIFFERENTIAL EXPLORATION OF COCHLEAR AND VESTIBULAR RECEPTORS BY INTERACTION OF ARTIFICIAL AND NATURAL STIMULI
}

\author{
R. Charlet DE SAUVAge, G. DOLIVET, J.-P. ERRE et J.-M. ARAN \\ Unité INSERM 229, Audiolọgie Expérimentale, Hôpital Pellegrin, F-33076 \\ Bordeaux, France
}

Résumé - On utilise une excitation électrique du VIIIème nerf combinée à une stimulation soit acoustique soit vestibulaire (bruit blanc ou accélération angulaire horizontale respectivement) pour extraire des réponses exemptes d’artẻfact,de spécificité soit cochléaire, soit vestibulaire. Chez le cobaye, la réponse Electrique-Vestibulaire (PAEV) apparaît comme une onde monophasique d'une amplitude de quelques dizaines de microvolts et d'une latence de l'ordre de $0,3 \mathrm{~ms}$, par opposition à la réponse électriquecochleaire (PAEC), couramment polyphasique et d'une amplitude de plusieurs centaines de microvolts. Schematiquement, le PAEV disparaît après destruction sélective des récepteurs vestibulaires (PAEC inchangé) alors qu'il se maintient après traîtement cochléotoxique (disparition đu PAEC), en accord avec les observations histologiques ultérieures. Les autres caractéristiques physiologiques des réponses confirment l'aptitude de la technique à dériver des composantes spécifiquement cochléaire et vestibulaire.

\begin{abstract}
An electrical excitation of the VIIIth nerve was used in combination with either an acoustic or a vestibular stimulus (white noise or horizontal angular acceleration respectively) to derive artefact-free responses with either cochlear or vestibular specificity. In the guinea pig, the Electrical-Vestibular response (EVAP) appeared to be monophasic with a few tens microvolts amplitude and a typical $0.3 \mathrm{~ms}$ latency, as opposed to the electrical-cochlear response (ECAP), a commonly polyphasic potential with a few hundreds microvolts amplitude. Schematically, the EVAP was abolished after a selective destruction of vestibular receptors (ECAP unchanged) while a cochleotoxic treatment left the EVAP unchanged (ECAP abolished), in accordance with further histological observations. Other physiological characteristics of the responses confirmed the ability of the technique to separate cochlear and vestibular specific components.
\end{abstract}

\section{1 - INTRODUCTION}

On sait que pour pouvoir observer la réponse composite d'un nerf il faut déclencher toutes les fibres instantanément, c'est-à-đire en moins d'l ms pour les fibres sensorielles. Avec une constante de temps de plusieurs secondes, le système vestibulaire ne remplit pas cette condition et il est impossible d'induire une réponse observable du nerf vestibulaire à l'aide d'accélérations situées dans la gamme physiologique. La stimulation électrique (SE) résoud le problème de la synchronisation des fibres et à ce titre a été souvent utilisée pour l'exploration des tissus nerveux. Cependant, deux inconvénients principaux limitent habituellement son application à des enregistrements en champ proche : 1) la SE est essentiellement aspécifique (une électrode sur la fenetre ronde peut, outre le VIIIème nerf, stimuler le nerf facial ou divers tissus musculaires), 2) elle engendre un artéfact électrique qui interfère avec la réponse physiologique et empêche l'observation des composantes de latence courte. Dans les années récentes notre équipe a pu résoudre ces difficultés pour le système cochléaire (1). Ainsi ont pu être enregistrées des réponses par combinaison de $\mathrm{SE}$ et de clics filtrés basse fréquence ou de sons continus (2), qui, seules, n'auraient pu fournir des réponses observable. Cet article décrit l'application du même principe au système vestibulaire et compare les réponses associêes à chacun des deux nerf cochleaire et vestibulaire.

\section{2 - METHODES EXPERIMENTALES}

Des cobayes tricoclores normaux ont été implantés au moyen d'électrodes sur la fenêtre ronđe et près du VIIIème nerf gauches, des électrodes indifférentes étant placées respectivement sur le vertex et le cervelet. Des SE transitoires de fréquence $100 \mathrm{~Hz}$ sont appliquées entre fenêtre ronde et vertex. Les enregistrements sont ef:ectués près du VIIIème nerf. Les stimulations vestibulaires consistent en des accélêrations angulaires, constantes ou type pendulaire, appliquées dans le plan du canal semi-circulaire horizontal gauche (3). La période d'acquisition peut être décalée par rapport aux instants de vitesse nulle afin d'explorer diverses phases du mouvement. Les stimulations acoustiques sont appliquées à l'aide d'un écouteur fixé latéralement sur la table tcurnante.

La technique, résumée sur la figure 1 , est fondèe sur une procédure différentielle décrite antérieurement $(1,4)$. Pour le système cochléaire, la SE étant appliquée en permanence, deux enregistrements sont effectués, l'un dans le silence (fig. la), l'autre en présence d'un bruit blanc masquant (fig. lb). Pour le système vestibulaire, le ler enregistrement est effectué au repos (fig. 1a), le second en présence d'une accélération angulaire (fig. 1b), et, pour éliminer l'effet éventuel du bruit engendré par la rotation, un bruit continu est présenté en permanence, à la fois pendant la rotation et pendant le repos. Sur les enregistrements de type a, on devine à peine la présence 
Stimulation électrique + ocoustique ou vestibuloire C $500 \mathrm{OG} / \mathrm{SE}=-0.60 \mathrm{~V} / \ddot{\mathrm{O}}=50^{\circ} \mathrm{s}^{-2} / \mathrm{BB} 80 \mathrm{~dB} \mathrm{SPL}$

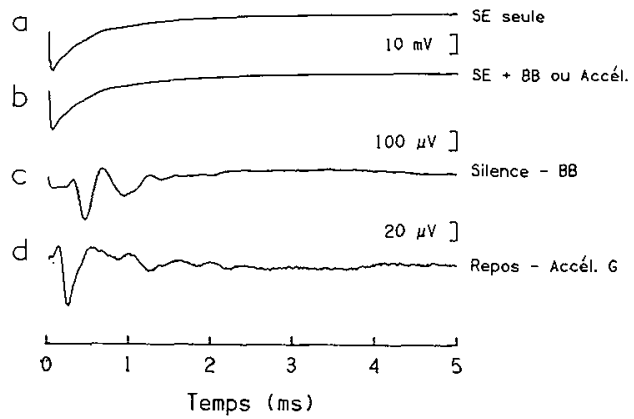

Fig. 1 - a) Artéfact + réponses physiologiques aspecifiques enregistrêes près du VIIIème nerf au cours d'une Stimulation Electrique (SE) constituée par une onde carrée appliquée sur la fenêtre ronde gauche de la cochlée d'un cobaye au repos. b) Enregistrement identique à " $a$ " mais soit en présence d'un bruit blanc masquant, soit au cours d'une accélération constante vers la gauche (dans ce dernier cas, un bruit est appliqué en permanence en a et $b$ afin d'eliminer toute contribution éventuelle d'origine cochléaire). c) Différence $a-b$ dans le cas du masquage cochléaire seul, laissant subsister la réponse dérivée cochléaire (PAEC, $2 \times 100$ passages). d) Différence $a-b$ dans le cas du mouvement rotatoire, laissant subsister la réponse dérivée vestibulaire (PAEV, $2 \times 100$ passages répété 5 fois). Comparer l'amplitude des deux réponses à celle de l'artéfact.

d'une réponse physiologique superposée à l'artéfact. Le bruit ou l'accélération inhibe tout ou partie de la réponse du nerf́ cochléaire ou vestibulaire à la SE (fig. 1b) sans modifier l'artéfact. Par différence entre les signaux a et $b$, l'artéfact s'élimine, ne laissant subsister que la réponse physiologique cochléaire (fig. lc) ou vestibulaire (fig. 1d). La procédure est répétée éventuellement plusieurs fois pour améliorer le rapport signal/bruit. La SE apporte la synchronisation nécessaire à l'observation d'une réponse alors que la stimulation cochléaire ou vestibulaire permet de dériver une réponse spécifique et exempte d'artéfact. Cette réponse, qui représente la variation d'excitabilité des fibres cochléaires ou vestibulaires due respectivement au bruit ou à la rotation, sera désignée sous le nom de Potentiel d'Action évoqué par stimulation Electrique et Cochléaire (PAEC) ou Vestibulaire (PAEV).

\section{3 - RESULTATS}

Les PAEV (fig. 2, colonne droite) présentent une forme essentiellement monophasique voisine de celle des PAEC (colonne gauche) quoique de latence légèrement plus courte $(0,3 \mathrm{~ms}$ au lieu de $0,4 \mathrm{~ms})$. La fonction de transfert des PAEC et des PAEV en fonction de l'intensité de stimulation acoustique ou vestibulaire apparaît globalement linéaire pour les intensités moyennes, les PAEC ayant une amplitude nettement supérieure à celle des PAEV. La polarité des PAEC apparaît negative pour la plupart des réponses dérivees dans les conditions "silence - bruit". De même les PAEV dérivés dans la condition "repos - accélération vers la gauche" sont en général négatifs (et positifs pour la condition "repos - accélération vers la droite). On observe parfois l'inverse chez certains cobayes, mais dans tous les cas les accélérations vers la gauche et vers la droite déterminent des réponses de polarité opposée entre elles chez le même animal.

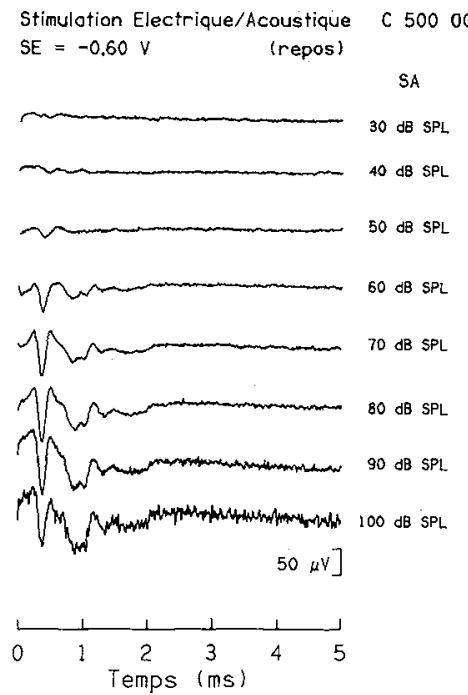

Stimulation Electrique/Vestibuloire $S E=-0.60 \mathrm{~V}$ (bruit permonent)

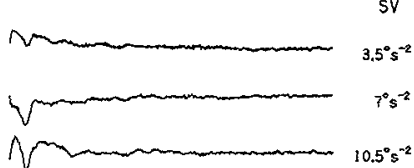

Fig. 2 - PAEC et PAEV dérivés comme indiqué sur la figure 1 , respectivement en fonction de l'intensité du bruit masquant (colonne gauche) et de l'amplitude de l'accélération (colonne droite). Les réponses cochléaires sont obtenues en soustrayant au signal moyenné dans le silence le signal moyenné en présence d'un bruit blanc (2x100 passages). Les réponses vestibulaires sont extraites pendant 5 périodes de stimulation pendulaire, en soustrayant alternativement des moyennes de 100 passages obtenues $1 \mathrm{~s}$ après l'accélération maximale à droite des enregistrements homologues à gauche $(5 \times 2 \times 100$ passages). Période du mouvement pendulaire $10 \mathrm{~s}$. 


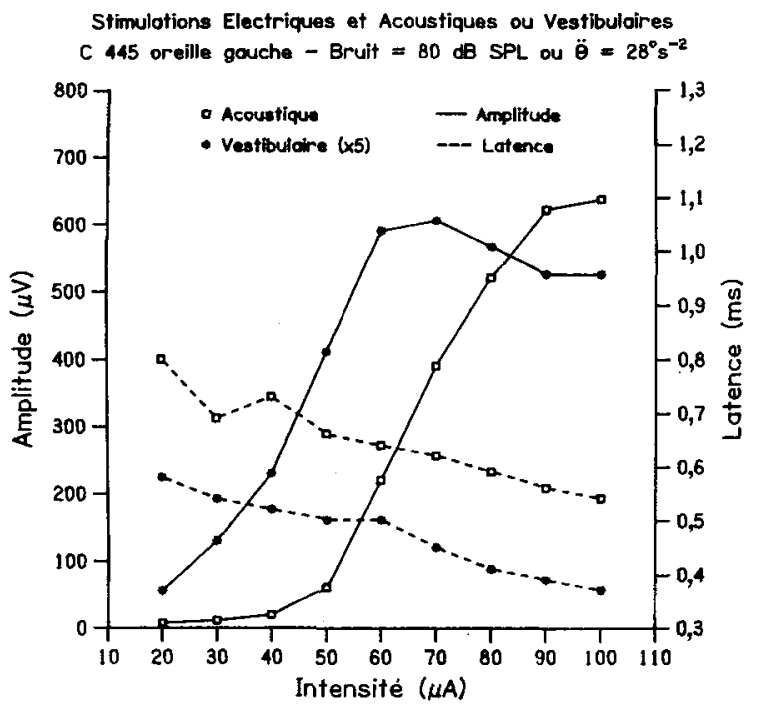

Fig. 3 - Influence de l'intensité de la SE (impulsions négatives de courant de durée $300 \mu \mathrm{s})$ sur l'amplitude (trait plein) et la latence (trait interrompu) des réponses cochléaires ( $\square$ ) et vestibulaires $\left(^{*}\right)$. Noter la similitude des seuils des réponses acoustiques et vestibulaires, ainsi que leur rapport d'amplitude.

L'influence de l'intensité de SE a également été étudiée (fig. 3). Les amplitudes relatives des PAEC et des PAEV présentent, dans leur portion linéaire, une croissance parallèle en fonction de l'intensité de SE. Par ailleurs la latence décroît de façon monotone en fonction de l'intensité.

Afin d'étudier plus en détail la spécificité du PAEV par rapport au PAEC, deux expériences utilisant des destructions sélectives des récepteurs cochléaires et vestibulaires ont été effectuées. Dans une première expérimentation, un cobaye préimplanté ayant initialement des fonctions cochléaire et vestibulaire normales a reçu une dose unique d'amikacine IM au taux de $500 \mathrm{mg} / \mathrm{kg}$, suivie d'une dose intracardiaque d'acide éthacrynique au taux de $50 \mathrm{mg} / \mathrm{kg}$. On sait qu'un tel traîtement est principalement cochléotoxique et produit une destruction complète de l'organe de Corti en quelques heures. On a pu effectivement vérifier chez cet animal que 4 heures après la dernière injection aucune réponse cochléaire n'était plus enregistrable: Au contraire, le PAEV était peu modifié à la fin de l'expérience (fig. 4, colonne gauche) confirmant l'origine non acoustique de la réponse. Dans une seconde expérience, un traîtement chronique a été entrepris à l'aide de streptomycine, antibiotique principalement vestibulotoxique. Les animaux ont reçu 21 injections IP 5 jours par semaine au taux de $200 \mathrm{mg} / \mathrm{kg}$. A la fin de l'expérimentation les potentiels cochléaires demeuraient normaux, alors que les PAEV avaient disparu à des degrés divers. Chez un cobaye particulier dont les réponses vestibulaires avaient totalement disparu (fig. 4, colonne droite), l'histologie a confirmé la présence de lésions importantes des organes vestibulaires, particulièrement les crêtes ampullaires, alors que les cellules cilièes cochleaires restaient normales.

\section{4 - DISCUSSION}

Les résultats présentés ici n'ont qu'un caractère illustratif et ne constituent pas une étude statistique des caractéristiques du PAEV.

La forme du potentiel d'action composite enregistré près d'une segment de nerf émergeant d'un milieu isolant a été analysée en détail par Lorente de Nò (5). Il a montré que cette forme pouvait changer de manière radicale selon la position longitudinale et radiale de l'électrode par rapport au nerf. Dans le cas présent, le nerf êmergeant du conduit auditif interne isolant reproduit les conditions d'expérimentation précédentes. Bien qu'on utilise une technique stéréotaxique pour l'implantation de l'électrode d'enregistrement, sa position par rapport au nerf est assez imprécise, à cause des variations anatomiques. Cela peut expliquer les variations de forme des réponses, y compris l'inversion de polarité observée chez certains animaux. Par ailleurs, l'amplitude relativement faible de la réponse vestibulaire par rapport à celle de la réponse acoustique (fig. 2, 3) peut s'expliquer par le nombre nettement plus faible des fibres vestibulaire impliquées, soit environ 2000 par canal semi-circulaire contre 24000 pour la cochlée (6).

La courbe d'amplitude du PAEC et du PAEV en fonction de l'intensité de SE présente une dynamique d'environ $10 \mathrm{~dB}$ (fig. 3). Les observations effectuées chez des sujets sourds équipés d'un implant cochléaire mettent en évidence une dynamique analogue allant de 6 à $20 \mathrm{~dB}$ (cf. 7, par exemple). La variation de latence des réponses en fonction de l'intensité du courant de SE est probablement due au temps d'établissement de la stimulation : à mesure que l'intensité augmente, le seuil de déclenchement des fibres est atteint de plus en plus tot, d'où une latence de plus en plus pécoce. 


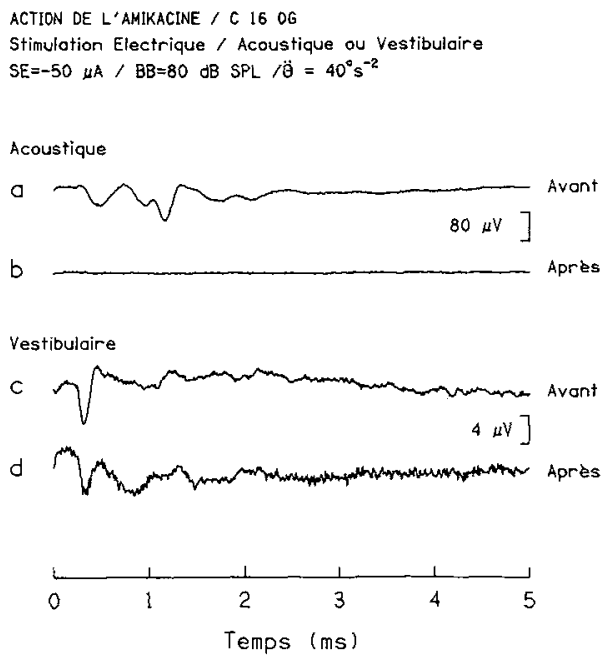

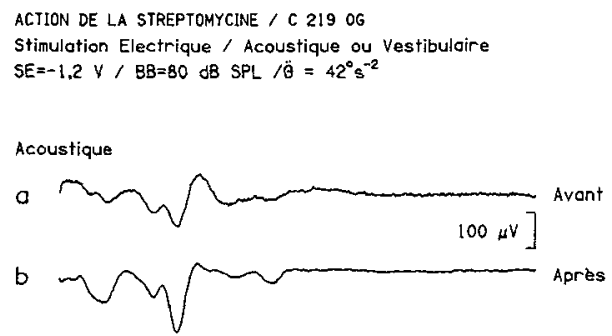
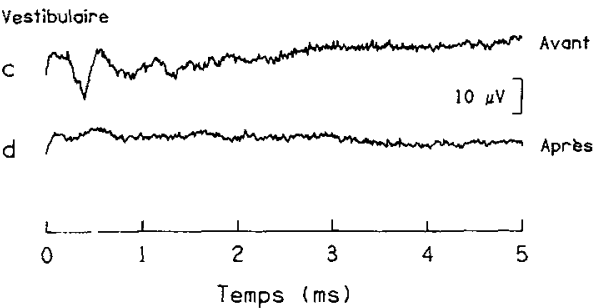

Fig. 4 - Comparaison des PAEC et des PAEV avant et après traitement par amikacine / acide éthacrynique (colonne gauche) et par streptomycine (colonne droite). Procédure identique à celle de la figure 1 ( $2 \times 100$ passages, répété 10 fois). Noter la disparition sélective du PAEC et du PAEV respectivement après le traitement cochléo et vestibulo-toxique.

On sait que les stimulations excitatrices (accélérations gauches dans le cas présent) augmentent le rythme de décharge spontané des fibres (8), réduisant sans doute en même temps leur excitabilité électrique. Dans cette hypothèse, accélération gauche et masquage acoustique contribueraient tous deux à réduire l'excitabilité électrique des fibres et devraient donner des réponses de même polarité, les accélérations droites donnant des réponses de polarité opposée. C'est effectivement ce que l'on observe. La relation quasi linéaire entre l'accélération et l'amplitude des PAEV peut être rapprochée de la caractéristique linéaire de la fonction de transfert liant le rythme de décharge des fibres des canaux semi-circulaires à l'accélération angulaire (9).

Les destructions vestibulaires et cochléaires sélectives confirment l'aptitude de la méthode à dériver des composantes spécifiquement cochléaires et vestibulaires. Si elle s'avère applicable aux enregistrements par électrodes de surface ou à partir de la fenêtre ronde, cette technique pourrait permettre d'élargir le champ des examens cliniques cochléaires (seuils objectifs aux fréquences graves), et pourrait également aboutir au développement d'examens objectifs de la fonction vestibulaire périphérique (Electrovestibulographie), venant en complément des méthodes d'exploration globales telles l'Electronystagmographie.

\section{REFERENCES}

/1/ Charlet de Sauvage, R., Cazals Y., Erre, J.-P. and J.-M. ARan. Acoustically derived auditory nerve action potentials evoked by electrical stimulation : an estimation of the waveform of single unit contribution. J. Acoust. Soc. Am. 73 (1983) 616-627.

12/ Aran, J.-M., ERre, J.-P. AND R. Chartet de Sauvage. Derived evoked potentials for continuous tones using a hybrid electrical-acoustical stimulation. Hearing Res. 20 (1985) 289-293.

13/ Curthoys, I.S., Curthoys, E.J,. Blanks, R.H.I. AND C.H.MarkHaM. The orientation of the semicircular canals in the guinea pig. Acta Otolaryngol. $\underline{80}$ (1975) 197-205.

/4/ R. Charlet de Sauvage, Erre, J.-P. AND Aran, J.-M. Electrovestibulogram : first results in the guinea pig. Acta Otolaryngol. (Stockh.) 107 (1989) 489-495.

/5/ LoRente De No, R. A study of nerve physiology. New-York Rockfeller Inst. Med. Res. (1947) two volumes 1044 pages.

/6/ GACEK, R.R. AND G.L. RASMUSSEN. Fiber analysis of the statoacoustic nerve of guinea pig, cat and monkey. Anat. Rec. 139 (1961) 455-463.

7/ House, W.F. AND J.URBan. Long-term results of electrode implantation and electronic stimulation of the cochlea in man. Ann. Otorhinolaryngol. 82 (1973) 504-517.

/8/ CuRThoYs, I.S., Blanks, R.H.I. and C.H. MARkHAM. Semicircular canal functional anatomy in cat, guinea pig and man. Acta Otolaryngol. $\underline{83}$ (1977) 258-265.

/9/ Goldberg, C. AND J.M. FERNANDEz. Physiology of peripheral neurons innervating semicircular canals of the squirrel monkey. 1. Resting discharge and response to constant angular accelerations. J. Neurophysiol. 34 (1971) 635-660. 